\title{
Ambulante Behandlung durch die psychiatrische Klinik
}

THOMAS SCHILLEN, MONIKA THIEX-KREYE

Priv.-Doz. Dr. med. Dipl. Phys. Thomas Schillen ist Chefarzt der Klinik für Psychiatrie und Psychotherapie, Klinikum Hanau GmbH

Dipl.-Betrw. Monika Thiex-Kreye, MBA ist Geschäftsführerin der Klinikum Hanau GmbH

\author{
Eine stationsersetzende, ambulante Akutbehandlung \\ durch die psychiatrische Klinik ist möglich. Sie \\ wird bisher nur durch die Zwänge bestehender \\ Finanzierungssysteme verhindert. Das OVP-Projekt \\ von AOK Hessen, Techniker Krankenkasse und Klinikum \\ Hanau (Lehmann et al. 2011) ermöglicht durch ein \\ integriertes Budget aller Behandlungssektoren die \\ Erprobung neuer Versorgungsformen der Klinik. \\ Im Rahmen dieses Projektes wurde inzwischen eine \\ Station mit 25 Betten geschlossen und durch ein \\ Team zur ambulanten Akutbehandlung abgelöst.
}

\section{Aktuelle Rahmenbedingungen der psychiatrischen Versorgung}

\subsection{Bedarf}

Seit 20 Jahren ist weltweit eine ungebrochene Zunahme der psychischen Störungen zu beobachten. Die Prognosen der WHO aus den 1990er Jahren werden durch ihre aktuelle Studie zur Global Burden of Disease 2010 bestätigt. Vergleichbare Ergebnisse wurden durch den Bundes-Gesundheitssurvey für die Versorgungssituation psychischer Störungen in Deutschland ermittelt. Auch die Gesundheitsreports der verschiedenen Krankenkassen stellen die erhebliche Zunahme psychischer Störungen fest. Allein bei der Anzahl der Arbeitsunfähigkeitstage durch psychische Erkrankungen findet sich eine Verdopplung während der letzten 10 Jahre. Dabei hat sich auch die Anzahl der betroffenen Patienten beispielsweise bei den depressiven Störungen mehr als verdoppelt.

\subsection{Defizite nach Versorgungssektoren}

Die steigenden Patientenzahlen haben zunehmende Defizite der psychiatrischen Versorgung zur Folge. Diese Defizite stellen sich in den Behandlungssektoren Kassenärztliche Versorgung (KV), Psychiatrische Institutsambulanz (PIA) und Psychiatrische Klinik unterschiedlich dar.

Im Bereich der KV wurde das Budget der niedergelassenen Psychiater der erheblichen Steigerung der Patientenzahlen nicht spürbar angepasst. Gleichzeitig ist die Anzahl der niedergelassenen Psychiater deutlich rückläufig, in manchen Regionen sogar verschwindend gering. Infolge dessen liegen die Behandlungszahlen in diesem Bereich bei bis zu 1000 Patienten pro Arzt und Quartal. Die Wartezeiten für einen regulären Behandlungstermin betragen teilweise 2 und mehr Monate.

Auch bei der psychotherapeutischen Versorgung im Bereich der KV weisen 
Wartezeiten von 6 bis 12 Monaten ebenfalls auf ein Versorgungsdefizit hin. Aufgrund der wöchentlichen Frequenz und einer Dauer der Therapie über Monate bis Jahre behandelt ein Psychotherapeut etwa 30 Patienten über einen längeren Zeitraum. Dabei entfallen etwa zwei Drittel des Psychiatrie- und Psychotherapie-Budgets der KV auf psychotherapeutische Leistungen.

In dieser Mangelsituation der kassenärztlichen Versorgung erweisen sich die psychiatrischen Institutsambulanzen der Kliniken als Rückfallebene für Hausund Fachärzte zur Klärung psychiatrischer Notfallindikationen. Aufgrund der kassenärztlichen Unterversorgung sind die Institutsambulanzen aber auch Anlaufstelle für die Selbstzuweisung durch die Patienten, die aufgrund ihrer psychischen Verfassung lange Wartezeiten im KV-Bereich nicht durchhalten. Aufgrund des Spezialauftrages der Institutsambulanzen nach $\mathbb{S} 118$ SGB V muss dabei jedoch ein größerer Teil dieser Patienten nach einer einmaligen Abklärung von Notfall- und Gefährdungsaspekten an den KV-Bereich zurückverwiesen werden. Im Ergebnis findet sich dadurch in den letzten Jahren eine deutliche Zunahme von Patienten mit Einmalkontakten in der PIA, die nicht angemessen weiterbehandelt werden dürfen. Für die PIA problematisch ist dabei auch die erneute Selbstzuweisung von Patienten, bei denen einzelne Krankenkassen die Vergütung einer erneuten Krisenintervention dann als unzulässige Weiterbehandlung strittig stellen.

Obwohl beispielsweise die Quartalspauschalen der PIAs in Hessen etwa viermal so hoch vergütet werden wie das Regelleistungsvolumen pro Patient im KV-Bereich, ergibt sich trotzdem ein Erlösdefizit der PIA bei Patienten, die mehr als 30 min Behandlungszeit pro Monat benötigen. Ein umfangreiches Assertive Community Treatment und aufsuchendes Home Treatment ist daher nur für einen kleinen Teil der PIA-Zielgruppe in einer Mischkalkulation über große Patientenzahlen finanziell darstellbar.

Im Behandlungssegment der psychiatrischen Klinik wirkt sich die unzureichende ambulante Versorgung als zunehmender Aufnahmedruck von Patienten aus, die nach Einschätzung der Klinik bei der stationären Aufnahme im Laufe der letzten Jahre zunehmend schwerer dekompensiert sind. Trotz wei- ter rückläufiger Verweildauern in der stationären psychiatrischen Behandlung bestehen in sehr vielen Kliniken seit Jahren Überbelegungen mit Auslastungen von deutlich über $95 \%$.

\subsection{Defizite nach psychiatrischer Störung}

Bei der Betrachtung der Versorgungsdefizite ist ferner nach der Art der psychischen Störung zu differenzieren.

Für einen größeren Teil der Patienten mit depressiven Störungen stellen antidepressive Medikation und strukturierte Kurzpsychotherapie erfolgreiche Behandlungsformen dar. Die Behandlungsmöglichkeiten in der kassenärztlichen Versorgung liegen hier aber weit hinter den Therapieempfehlungen der WHO zurück. Kurzinterventionen für episodische Störungen stehen nicht zeitnah und nicht im erforderlichen Umfang zur Verfügung. Hinzu kommt, dass immer noch ein großer Teil dieser psychischen Erkrankungen in der Primärversorgung nicht erkannt wird. Im Ergebnis erhalten dann weniger als $10 \%$ der Patienten eine leitliniengerechte Behandlung.

Während bei der Depression in vielen Fällen die zeitnahe adäquate Kurzintervention im Fokus steht, benötigen Menschen mit sehr schweren und/oder chronischen psychischen Erkrankungen häufig eine langfristige, teilweise jahrelange professionelle Begleitung. Dies trifft beispielsweise auf Patienten mit schizophrenen Psychosen oder auch mit chronischer Suizidalität bei schweren Persönlichkeitsstörungen nach Missbrauchserfahrung zu. Das Defizit der Versorgung besteht hier in häufigen Behandlungsabbrüchen im ambulanten Versorgungssektor. Mangelnde Krankheitseinsicht und fragliche Compliance der Patienten bei der Behandlung erfordern eine nachgehende und aktiv aufsuchende Begleitung und Vernetzung mit dem sozialen und professionellen Umfeld des Patienten. Die Versorgungsdefizite führen bei dieser Patientengruppe dann schnell zu Krankheitsverläufen mit häufigen Klinikeinweisungen als psychiatrische Notfälle durch die Polizei oder den gesetzlichen Betreuer.

\subsection{Defizite bei Personalgewinnung und Personalressourcen}

Die aktuelle Situation bei der Verfügbarkeit der verschiedenen Berufsgruppen im psychiatrischen Krankenhaus stellt sich dramatisch dar. Von den etwa 4000 Ärzten, die pro Jahr eine Facharztweiterbildung aufnehmen, geht nur $1 \%$ in die Psychiatrie. Die psychischen Erkrankungen werden als am Schwierigsten zu behandeln eingeschätzt. Die sehr hohe psychische Belastung des Personals durch die täglichen Notfallsituationen mit eigen- und fremdgefährdenden $\mathrm{Pa}$ tienten auf psychiatrischen Stationen erscheint wenig attraktiv. Diese Situation stellt sich für die große Gruppe der psychiatrischen Pflegekräfte vergleichbar dar.

Die zunehmende Leistungsverdichtung auch im psychiatrischen Krankenhaus mit zahlreichen Kriseninterventionen, kurzen Verweildauern und hohem Schnittstellenaufwand bei Auf-

\section{Die zunehmende Leistungsverdichtungen im psychiatrischen Krankenhaus haben zu einer grenzwertigen Verknappung der Personal- ressourcen pro Patient geführt.}

nahme und Entlassung haben zu einer grenzwertigen Verknappung der Personalressourcen pro Patient geführt. Das geplante neue Entgeltsystem mit einer Leistungserfassung von 25 MinutenEinheiten stellt sich in dieser angespannten Situation als nicht nachvollziehbare Verschwendung von Personalressourcen dar. Auch der neue Entwurf versteckter Fallpauschalen bessert diese Situation nicht.

\section{Zwänge der bestehenden Vergütungssysteme}

Die Gründe der skizzierten Versorgungsdefizite liegen zumindest zu einem großen Teil in der fehlenden Durchlässigkeit der verschiedenen Behandlungssektoren ambulanter und stationärer Versorgung.

Aus Sicht des Krankenhauses erscheint die von Sozialleistungsträgern gern bemühte Formulierung von „Fehl- 
anreizen zur Belegung stationärer Betten" nach BPflV oder auch nach neuem Entgeltsystem mit der Unterstellung behaftet, die steigenden Kosten der psychiatrischen Versorgung seien dem Umstand geschuldet, dass die Kliniken der Versuchung dieser Fehlanreize erliegen würden.

Zutreffend ist bisher jedoch, dass Krankenkassen und Kliniken Verträge über jährliche Budgets von Berechnungstagen vereinbaren müssen. Die Sozialleistungsträger kaufen beim Krankenhaus stationäre Behandlungstage für ihre Versicherten und finanzieren die für eine aufwendige psychiatrische Versorgung erforderlichen Ressourcen bisher auch überwiegend nur in diesem Sektor. Als Konsequenz stehen die Behandlungsangebote für die überwiegende Anzahl von Patienten mit einem höheren Versorgungsbedarf auch nur im Krankenhaus zur Verfügung.

Wie ausgeprägt diese Zwänge des Vergütungssystems sind, skizziert Abbildung 1: von 30 min alle 4 Wochen ausreichend behandelbar ist, erlöst in Hessen eine durchschnittliche Quartalspauschale von etwa $180 €$. Im täglichen Mittel

\section{Die Reduzierung der Kosten pro Patient in einem einzelnen Behandlungssektor führt nicht zwangsläufig zu einem Optimum des gesamten Systems.}

entspricht dies einer Behandlung von 1 min für $2 €$. Benötigt ein Patient der PIA bei Exazerbationen seiner Erkrankung einen Behandlungsaufwand von mehr als $1 \mathrm{~min}$ pro Tag, dann ist dies in der PIA für eine eng begrenzte $\mathrm{Pa}$ tientenzahl und Behandlungsdauer als Mischkalkulation vielleicht noch darstellbar. Über die gesamte Kohorte aller Patienten der PIA betrachtet bedeutet allerdings bereits die Verdoppelung des Behandlungsaufwands auf täglich

\section{Abbildung 1: Finanzierbarer Versorgungsbedarf nach Behandlungssektoren.} KV: Kassenärztliche Vereinigung, PIA: Psychiatrische Institutsambulanz.

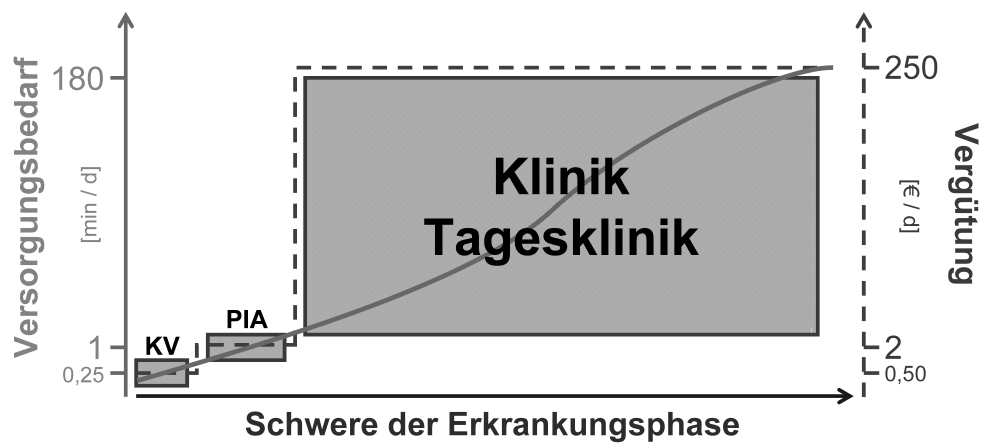

Der individuelle Behandlungsbedarf eines Patienten variiert abhängig von der aktuellen Schwere seiner Erkrankungsphase. Um die Therapiemöglichkeiten der verschiedenen Sektoren ambulanter und stationärer Versorgung vergleichen zu können, ist der Behandlungsbedarf in Behandlungsminuten pro Tag dargestellt (Durchgehende Kurve). Dem gegenüber zeigt die unterbrochene Kurve die Erlösbereiche der verschiedenen Versorgungssektoren in Erlös pro Tag.

Ein Patient der psychiatrischen Institutsambulanz (PIA), dessen aktuelles Zustandsbild mit einem Termin
2 min, dass die dafür erforderlichen Ressourcen nicht mehr im ambulanten, sondern nur noch im (teil)stationären Rahmen kostendeckend finanziert sind. Der Wechsel des Versorgungssektors von ambulant nach stationär wird dadurch für eine relevante Größenordnung der Patientenzahlen durch die Zwänge der Vergütungsstrukturen und nicht durch die fachlichen Behandlungsnotwendigkeiten erzwungen.

Eine vergleichbare Betrachtung ergibt übrigens für die KV-Versorgung durch den niedergelassenen Psychiater eine im Mittel kostendeckend mögliche
Behandlungsintensität von etwa $15 \mathrm{sec}$ für $0,50 €$ pro Tag.

Bei dieser Betrachtung der finanziellen Zwänge des Versorgungssystem ist allerdings auch zu ergänzen, dass die alleinige PIA-Behandlung für einen großen Anteil der Patienten hinreichend ist. Etwa $60 \%$ aller $\mathrm{Pa}$ tienten der PIA eines Behandlungsjahres sind ambulant so ausreichend versorgt, dass eine stationäre Versorgung nicht erforderlich wird. Bei den übrigen Patienten, deren Erkrankungsschwere phasenweise die Ressourcen der stationären Versorgung erforderlich macht, ist eine ambulante Behandlung in den bestehenden Finanzierungsstrukturen dagegen bisher in der Mehrzahl nicht möglich.

Bei den Bemühungen, die Kosten psychischer Störungen im Gesundheitssystem zu begrenzen, führt die Reduzierung der Kosten pro Patient in einem einzelnen Behandlungssektor eben nicht zwangsläufig zu einem Optimum des gesamten Systems. Hinzu kommt aus ärztlicher Sicht der Aspekt, dass die Aufnahme auf eine psychiatrische Station für viele Menschen doch immer noch eine erhebliche Hürde darstellt. So ist davon auszugehen, dass die unzureichende Bereitstellung ambulanter Ressourcen dazu beiträgt, dass zielführende störungsspezifische Kurzinterventionen dem Patienten nicht zeitnah zur Verfügung stehen und dadurch mittelfristig ein deutlich höherer Behandlungsaufwand mit auch schlechteren Behandlungsergebnissen für einen exazerbierten und chronifizierten Krankheitsverlauf entsteht.

\section{Lösungsansätze im Rahmen des Projektes zur Optimierten Versorgung in der Psychiatrie (OVP) von Techniker Krankenkasse, AOK Hessen und Klinikum Hanau}

\subsection{Ansätze des OVP-Vertrages}

Vor dem Hintergrund der dargestellten Probleme in der psychiatrischen Versorgung, die durch das geplante neue Entgeltsystem nicht gelöst, sondern aggraviert werden, verhandelte eine Arbeitsgruppe der Techniker Krankenkasse, der AOK Hessen und des Klinikums Hanau einen Lösungsansatz zur Erprobung neuer Versorgungsformen (Lehmann et al. 2011). 
- Im Expertenkreis besteht weitgehend Konsens darüber, dass ein relevanter Anteil stationärer Patienten stationsersetzend ambulant versorgt und/ oder früher aus der stationären Behandlung entlassen werden könnte, wenn diesen Patienten der individuell erforderliche, hohe Behandlungsbedarf im ambulanten Bereich angeboten werden könnte. Diese Einschätzung korrespondiert zu den unter 2 . dargestellten Überlegungen zu den Zwängen der getrennten Vergütungssysteme. Um hier neue Möglichkeiten einer allein vom Versorgungsbedarf des Patienten bestimmten Wahl des Behandlungssektors zu schaffen, wurde für das Projekt das anteilige Budget der Techniker Krankenkasse und der AOK Hessen aus den Bereichen PIA, Tagesklinik und Klinik in ein einziges OVP-Budget integriert. Innerhalb dieses garantierten OVPBudgets erhält die Klinik volle Flexibilität bei der Art der Leistungserbringung.

- Es bestand der Anspruch, ein Vergütungsmodell mit voller Kompatibilität zu den Vorgaben des $\mathbb{S} 17 \mathrm{~d}$ KHG zu entwickeln. Ausgehend von den Erfahrungen bei den somatischen DRGs, bei denen 90\% der Kalkulationshäuser fallbezogene Personalkosten anhand der PPR aufschlüsseln, wurden Kosten pro Tag, Patient und Behandlungskategorie (OPS 9-98) auf der Grundlage der PsychPV berechnet. Die daraus abgeleiteten tagesgleichen Relativgewichte ergeben in Verbindung mit dem vereinbarten Gesamtbudget den Tagesbasiswert der Klinik (Lehmann et al. 2011).

- Der Rückgriff auf Relativgewichte nach PsychPV-Einstufungen basiert zusätzlich auf der Überlegung, Erfassungsaufwand, Kodierfehler, MDKPrüfaufwand und Fehlanpassungen des Systems durch die Verwendung einer langjährig etablierten Kenngröße zu minimieren. Dies schont die Personalressourcen auf Seiten der Klinik und der Krankenkassen. Für die Einstufung der Relativgewichte wurden Überprüfungen durch den MDK umfangreicher zu Projektbeginn und im weiteren in Stichproben zweimal pro Jahr vereinbart.

- Die Relativgewichte der Institutsambulanz wurden zunächst mit $25 \%$ der stationären Relativgewichte geschätzt
(Lehmann et al. 2011). Die kontinuierliche Evaluation der ambulanten Relativgewichte ist Teil des Projektes. Dabei wird auch davon ausgegangen, dass sich durch die Flexibilisierung der Leistungssektoren neue ambulante Akutbehandlungen entwickeln werden, die in den Leistungskennzahlen der bisherigen PIA nicht enthalten sein können.

- Für die angestrebte Flexibilisierung der Leistungserbringung durch die Klinik ist der weitere Ausbau der Vernetzung mit den verschiedenen Leistungserbringern außerhalb der Klinik wesentlich. Zu Projektbeginn wurden hierzu die Hanauer LebensGestaltung (Tagesstätten, Betreutes Wohnen, Wohnheim, Beschütztes Arbeiten) und das Medizinische Versorgungszentrum Hanau als Kooperationspartner in das Projekt integriert.

- Fachlich basiert das Projekt auf einem Behandlungsmanual zum Vertrag einschließlich Qualitätskriterien für die Versorgung.

- Während der Laufzeit des Vertrages verzichtet das Klinikum Hanau darauf, seinen Anspruch auf eine Aufstockung der Bettenkapazität umzusetzen.

Das Projekt basiert aktuell auf einem IV-Vertrag nach $\mathbb{S} 140$ a SGB V zwischen Techniker Krankenkasse, AOK Hessen und Klinikum Hanau. Die Erweiterung auf alle Krankenkassen wäre im Sinne derjenigen Patienten, die bisher von den neuen Versorgungsformen ausgeschlossen werden müssen, sehr erstrebenswert. Auch eine Umwandlung in ein Modellprojekt nach dem neuen $\mathbb{S} 64 \mathrm{~b}$ SGB V erscheint sinnvoll, um den Aufwand und die Hürde einer Einschreibung in den IV-Vertrag zu beseitigen.

Das mit der AOK Hessen und der Techniker Krankenkasse vereinbarte OVP-Budget entspricht dem anteiligen, bisherigen Budget des Krankenhauses in den verschiedenen Leistungssektoren. Es enthält keine zusätzlichen Finanzierungsanteile und beinhaltet keinen Eingriff in andere Entgeltsysteme. Bei in den IV-Vertrag eingeschriebenen Patienten erfolgt die Rechnungslegung auf der Basis von Relativgewichten nach taggenauen OPS 9-98 einschließlich deren
Erweiterung auf die PIA (Lehmann et al. 2011). Für die übrigen Patienten der AOK Hessen und der Techniker Krankenkasse wird die bisherige Abrechnungsform nach BPflV beibehalten. Im

\section{-Vergütung personal- er Leistungen möglicht eine bedarfs- chte Individualisierung des diungssektors.}

Rahmen der jährlichen Anpassung der Relativgewichte an die sich entwickelnden Leistungsprofile kann das bisherige stationäre Budget der Entwicklung stationsersetzender, ambulanter Leistungen folgen.

\subsection{Ansätze für die Patientenversorgung}

Aus Sicht des Arztes ist das vorrangige Vertragsziel die optimierte Versorgung der Patienten mit den verfügbaren Ressourcen. Die OVP-Vergütung personalintensiver Leistungen unabhängig vom stationären Bett ermöglicht eine bedarfsgerechte Individualisierung des Behandlungssektors ohne die Zwänge der bisherigen BPflV oder auch des geplanten Entgeltsystems der Psychiatrie. Typische Behandlungselemente des Assertive Community Treatsments, die in der Institutsambulanz seit langem etabliert sind, können damit allen Versicherten der AOK Hessen und der Techniker Krankenkasse angeboten und weiterentwickelt werden. Die Behandlung lässt sich dadurch besser an die Bedürfnisse des Patienten und seines sozialen Umfeldes anpassen. Hochfrequente ambulante Kontakte in der Klinik, aufsuchende Behandlungen zu Hause oder im Wohnheim werden für einen Teil der Patienten die stationäre Versorgung ersetzen können.

Auch der aktuelle Verzicht des Klinikums, den Anspruch auf eine Aufstockung der stationären Betten umzusetzen, ist durch das Ziel einer verbesserten Patientenversorgung mit den verfügbaren Personalressourcen motiviert. Die Erfahrungen in anderen Ländern der EU (Skandinavien, England) sowie einzelner Kliniken in Deutschland (Itzehoe, 
Geesthacht u.a.) legen die Konzentration auf ambulante Versorgungskonzepte nahe. In Anbetracht der anhaltend steigenden Patientenzahlen erscheint die Ausweitung ambulanter Behandlungen durch das Krankenhaus gegenüber einem Ausbau der stationären Kapazitäten vorrangig.

Für Patienten mit schweren rezidivierenden und/oder chronischen Erkrankungen stellt die langfristige Begleitung und stabile Beziehungskonstanz eine wesentliche Voraussetzung für den Behandlungserfolg dar. Therapieforschung und klinische Erfahrung belegen die Bedeutung einer tragfähigen Behandlungsbeziehung als Prädiktor für das Behandlungsergebnis. Im OVP-Projekt wird dem Patienten deshalb ein Bezugsbegleiter (Fallmanager) der Klinik als koordinierender Ansprechpartner für den Patienten, sein soziales Umfeld und die Leistungserbringer im Netzwerk zur Seite gestellt. Ziel ist eine verbesserte Integration der Behandlungsprozesse, die Reduzierung von Schnittstellenbrüchen und die Vermeidung von Behandlungsabbrüchen. Die dafür erforderlichen Ressourcen können je nach Patient und Störungsbild sehr erheblich sein. Unsere Erfahrungen mit einzelnen Patienten der PIA sowie in einem Pilotprojekt einer Station, die in den letzten Jahren bereits nach diesem Konzept behandelt wurden, zeigen, dass mittelfristig eine Stabilisierung des Patienten erreicht werden kann, in deren Folge eine deutlich reduzierte stationäre Behandlungsbedürftigkeit festzustellen ist. Darüber hinaus wurde nach subjektiver Einschätzung ein so gutes Behandlungsergebnis erreicht, wie es in der vorhergehenden Krankengeschichte nicht möglich war. Die Ausweitung dieser Behandlungsansätze auf große Patientenzahlen und alle psychischen Störungsbilder im Rahmen des OVP-Projektes wird in den nächsten Jahren zeigen müssen, in welcher Weise sich diese positiven Vorerfahrung auf die psychiatrische Pflichtversorgung einer ganzen Region übertragen lassen.

\section{Umsetzung des OVP- Projektes am Klinikum Hanau}

\subsection{Erste Schritte auf allen Stationen}

In einer ersten Projektphase wurden auf drei Stationen jeweils 2 Betten geschlossen und die betreffenden Räume für ambulante OVP-Leistungen auf Station verfügbar gemacht. Jeder Station wurden 2 Ärzte und 1 Pflegemitarbeiter der Institutsambulanz als Mentoren zugeordnet. Oberarzt und Pflegeleitung supervidierten wöchentliche OVPBehandlungsplanungen mit dem Team der Station.

Initial wurden primär versucht, Intensivnutzer der Stationen als erste OVP-Patienten zu gewinnen. Deren Bezugsbegleiter im Projekt berichteten über ihre neuen Erfahrungen mit diesen Patienten in ihrem sozialen Umfeld. Die Vorteile und erste Erfolge der ambulanten Beziehungskonstanz wurden für die Mitarbeiter erlebbar.

Für den Prozess der OVP-Einschreibung wurde eine standardisierte Erhebung des sozialen und professionellen Umfeldes des Patienten festgelegt. Dadurch können die langfristig tragenden Beziehungen des Patienten in die Begleitung integriert und unterstützt werden, ohne Doppelstrukturen aufzubauen.

Das gesamte Therapieangebot der Abteilung mit etwa 130 Gruppentherapien wurde neu auf ein durchgängiges, gemeinsames Zeitraster aller ambulanten, teil- und vollstationären Behandlungseinheiten geordnet. Die Gruppenangebote sind dadurch grundsätzlich für alle Behandlungssektoren nutzbar. Ein Patient, der zwischen ambulanter und stationärer Versorgung wechselt, kann damit je nach Indikation weiter an übergreifenden Gruppen teilnehmen.

Die stationären Gruppenangebote stehen OVP-Patienten auch ambulant zur Verfügung. Im Rahmen des Projektes wurden eine Reihe zusätzlicher Gruppenangebote eingeführt. Mitarbeiter der stationären Behandlungsteams begannen mit Hausbesuchen bei ausgesuchten OVP-Patienten. Die PIA reservierte zusätzliche Notfallsprechstunden für ambulante Kriseninterventionen bei OVP-Patienten.

Neben dem Wissenstransfer aus der PIA und dem Pilotprojekt in alle stationären Behandlungsteams wurde eine 2-jährige InHouse-Weiterbildung in Systemischer Therapie für 22 Mitarbeiter aller Berufsgruppen in allen Behandlungseinheiten begonnen.

\subsection{Fallvignetten zu ersten Erfolgen}

Ein 65-jähriger Patient mit einer langjährig chronischen, schizophrenen Psychose fühlt sich in den letzten 5 Jahren zunehmend durch Bestrahlungen in seiner Wohnung und auch auf Station gequält. Es gibt kaum symptomfreie Intervalle. Der Patient zieht sich immer mehr in seine Wohnung zurück und lebt sozial völlig isoliert.

In den letzten 2 Jahren kommt es zu zunehmend häufigeren, insgesamt 7 stationären Aufenthalten von jeweils 1 - 3 Monaten. Es besteht keine medikamentöse Compliance. Ein ambulanter Pflegedienst zur täglichen Medikation wird regelmäßig wenige Tage nach der stationären Entlassung nicht mehr in die Wohnung gelassen. Bald darauf erfolgt dann nach Dekompensation die Wiederaufnahme als Zwangseinweisung.

Als OVP-Patient lässt der Patient Hausbesuche seines Bezugskrankenpflegers der Station alle 2 Wochen zu. Er lässt sich auf eine Depotmedikation ein. Im Rahmen der Beziehungsarbeit gibt der Patient nach einem halben Jahr seine Vorbehalte gegen den Umzug in ein betreutes Wohnheim auf. Zwei Monate nach seinem Umzug fühlt der Patient in seinem neuen Zimmer keine Bestrahlung mehr. Er nimmt mit Freude an Freizeitgruppen der Einrichtung teil. Die Überleitung in die Weiterversorgung nach SGB XII ist gelungen und das Krankenhaus kann die Intensität seines Behandlungsangebots wieder reduzieren.

Eine schwer traumatisierte Borderline-Patientin kommt in den letzten Jahren zu zahlreichen und zunehmenden stationären Behandlung von teilweise monatelanger Dauer. Während dieser Aufenthalte führt die Patientin auch im stationären Rahmen zahlreiche Intoxikationen herbei, die immer wieder auch eine Intervention auf der Intensivstation erforderlich machen. Im Rahmen des OVP-Projektes gelingt der Bezugskrankenschwester die Gratwanderung zwischen Autonomiebedürfnis der Patientin und supportivem Halt durch den vertrauten Rahmen des Stationsteams. 
Die Patientin besucht die Station zu täglichen, ambulanten Kontakten, auch am Wochenende, bei denen sie ihre Medikation erhält. Es kommt zu einer deutlich besseren Stabilisierung der Patientin als während der stationären Behandlungen.

Eine Patientin mit schizoaffektiver Psychose benötigt in einem Zeitraum von 10 Jahren insgesamt $2 \frac{1}{2} 2$ stationäre Behandlungsjahre bei etwa 25 Klinikaufenthalten. Die Einweisungen erfolgen in aller Regel als Zwangseinweisungen bei schizomanischer Dekompensation. Als OVP-Patientin meldet sie sich bei einer erneuten Verschlechterung in Eigeninitiative bei ihrer Bezugsärztin.

\subsection{Schließung einer Station, Einführung eines OVP-Teams zur ambulanten Akutbehandlung}

Ein Jahr nach dem Beginn des OVPProjektes wurden die bisherigen OVPErfahrungen der Stationen in einem eigenständigen Team zur stationsersetzenden, ambulanten Akutbehandlung zusammengeführt. Dieses OVP-Team hat den Auftrag, Versicherte der Techniker Krankenkasse und der AOK Hessen, die aufgrund der Schwere ihrer psychischen Erkrankung bisher stationär aufgenommen werden mussten, nach Möglichkeit mit einem intensiven Therapieangebot im individuell erforderlichen Umfang (need-adapted treatment, assertive community treatment) ambulant $\mathrm{zu}$ behandeln. Bei Patienten, bei denen die stationäre Aufnahme zunächst unvermeidlich ist, trägt das OVP-Team durch engmaschige Kontaktaufnahme mit dem Patienten und der Station dazu bei, den stationären Aufenthalt durch die Übernahme des Patienten in die ambulante Akutbehandlung zu verkürzen.

Nach ausführlicher Information der Mitarbeiter wurde die Mitarbeit im OVP-Team abteilungsintern ausgeschrieben und ein Team von etwa Stationsstärke zusammengestellt: 1 VK Oberarzt, 2 VK Assistenzärzte, 0,5 VK Psychologin, 13 VK Pflege, 1 VK Sozialarbeiter, 1 VK Ergotherapie, Anteile Bewegungstherapie, 1 VK Stationsassistentin. Die Aufstockung der pflegerischen Berufsgruppe erfolgt gestuft im Rahmen eines Übergangsplans und abhängig von der Entwicklung der Ambulantisierung.

Am 31.05.2012 wurde die bisherige Depressionsstation mit 25 Betten

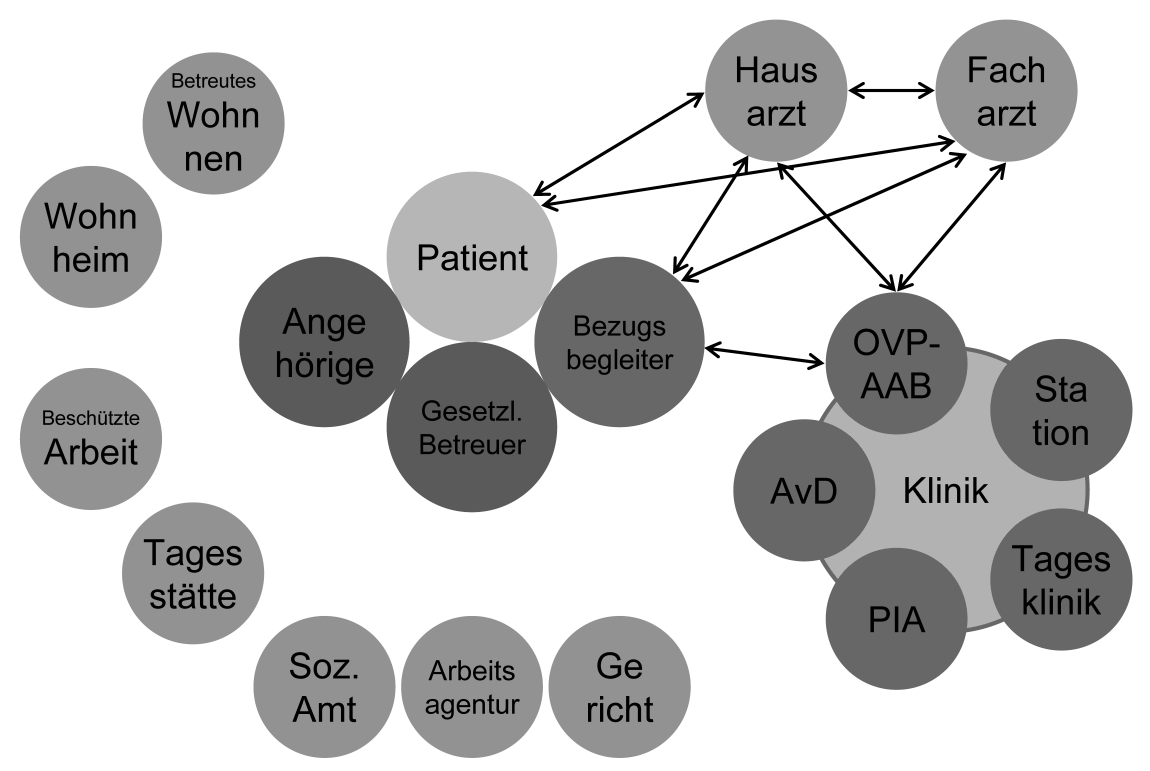

Abbildung 2: Ambulante Behandlung durch die Klinik im psychiatrischen Netzwerk. OVP-ABB: Ambulante Akutbehandlung im OVP-Projekt, PIA: Psychiatrische Institutsambulanz

geschlossen und ab 01.06.2012 in den Räumlichkeiten dieser Station das Team zur ambulanten Akutbehandlung eingeführt. Bei der Schließung einer unserer Stationen fiel die Wahl auf die Depressionsstation, da gerade die dort behandelten Patienten auch bei schwerer Depression überwiegend verlässlich absprachefähig sind. Als Kontraindikationen für eine ambulante Akutbehandlung dieser Patienten definierten wir akute Suizidalität, wahnhafte Depression und schweren Antriebsverlust von einem Ausmaß, das den Patienten am täglichen Aufsuchen der Klinik hindert. Etwa die Hälfte der anwesenden Patienten der Depressionsstation waren Versicherte der AOK Hessen und der Techniker Krankenkasse. Von diesen konnten 8 Patienten unmittelbar aus der stationären Versorgung in die ambulante Akutbehandlung übernommen wer-

Neue Versorgungsformen, wie die ambulante Akutversorgung, bedürfen einer Überprüfung der Behandlungsqualität.

den. Die übrigen Patienten der Depressionsstation wurden auf die verbleibenden 3 Stationen verlegt. Die erforderlichen Kapazitäten wurden dadurch geschaffen, dass eine korrespondierende Anzahl an Patienten von den Stationen in die ambulante Akutbehandlung entlassen wurde. Aktuell befinden sich zwischen 30 und 35 Patientin in der ambulanten Akutbehandlung. Die Zuweisung erfolgt über die Stationen, die PIA, Hausärzte oder Fachärzte.

Die ambulante Akutbehandlung durch das OVP-Team stellt damit ein neues Element im ambulanten Behandlungsangebot der Klinik dar (Abbildung 2: OVP-AAB), dessen Einführung im vollen Behandlungsvolumen einer ganzen Station erst durch die Flexibilität des OVP-Vertrages realisierbar wurde.

\section{OVP Perspektiven}

\subsection{Was zu bedenken ist}

In dem hier vorgestellten integrierten Budget fehlen die ambulanten Leistungen der KV. An der Schnittstelle $\mathrm{zu}$ anderen Finanzierungssystemen entstehen leicht Verwerfungen und Begehrlichkeiten. Es ist deshalb zu beachten, dass der Klinik im OVP-Budget genau die Personalressourcen der bisherigen Leistungen zur Verfügung stehen. Ambulantisieren lassen sich daher auch nur die Leistungen, die bisher stationär erbracht wurden. Wenn langfristige Effekte einer besseren Stabilisierung chronisch kranker Intensivnutzer zu erreichen sind, erscheint eine Ausweitung der Patientenzahlen im Umfang dieser 
Effekte möglich. Die einleitend dargestellten Versorgungsdefizite der kassenärztlichen Versorgung können durch die Flexibilisierung des Klinikbudgets allerdings nicht ausgeglichen werden. Die von niedergelassen Hausärzten dringend angefragte, regelmäßige gemeinsame gerontopsychiatrische Visite im Altenheim ist fachlich sinnvoll, in diesem Rahmen aber nicht darstellbar. Auch Therapieprogramme zur ambulanten Suchtbehandlung wie ALITA mit nachweislich sehr erfolgreichen, nachhaltigen Abstinenzergebnissen lassen sich nicht im Rahmen des verfügbaren Budgets umsetzen, da sie nach Art, Umfang und Dauer ganz erheblich über die bisherigen Alkoholentgiftungen im stationären Kontext hinausgehen.

Ebenfalls zu bedenken ist, dass die in einigen Regionen zwischen einzelnen Krankenkassen und sozialpsychiatrischen Vereinen geschlossenen IV-Verträge, die neue ambulante Versorgungsangebote dieser Vereine mit Malus-Regelungen für stationäre Behandlungen verbinden, das Kernproblem der kassenärztlichen Unterversorgung ebenfalls nicht lösen. Stattdessen schaffen diese IV-Verträge Anreize für die Entwicklung von Doppelstrukturen parallel zu den Versorgungsangeboten der Klinik und zu Lasten von SGB V-Budgets - eine sowohl fachlich als auch wirtschaftlich fragwürdige Entwicklung.

\subsection{Weitere Entwicklung}

Ambulante Versorgung ist daher nicht schon einfach deshalb gut, nur weil sie ambulant erfolgt. Neue Versorgungsformen wie die ambulante Akutversorgung bedürfen einer Überprüfung der Behandlungsqualität (HoNOS). Eine bessere therapeutische Beziehung und Stabilisierung gerade bei schwer und chronisch psychisch kranken Menschen ist nicht durch deren Teilnahmeerklärung bei einem Projekt zu erreichen. Es geht vor allem um eine langfristige Beziehungsarbeit mit dem Patienten sowie eine engere Vernetzung mit seinem sozialen und professionellen Umfeld, um eine erfolgreiche Begleitung zu ermöglichen.

Ambulante Versorgung ist auch nicht einfach schon deshalb kostengünstig, nur weil sie ambulant ist. Bei chronisch kranken Intensivnutzern einer Klinik mit hohen Wiederaufnahmeraten kann der ambulante Aufwand für die Arbeit mit dem Patienten und seinem Umfeld sehr erheblich sein. Gerade bei dieser Patientengruppe erscheint dieser Ansatz jedoch als der richtige, mit langfristig wahrscheinlich besserer Lebens- und Behandlungsqualität. Die Beurteilung der wirtschaftlichen Aspekte erfordert dabei eine Analyse über die nächsten Jahre und größere Patientenzahlen.

Die Versorgung der großen Gruppe depressiver Patienten könnte sich dagegen ganz anders darstellen. Nicht wenige Patienten erleben die Aufnahme auf einer psychiatrischen Station immer noch als stigmatisierend und bedrohlich. Viele Patienten lehnen eine stationäre Aufnahme so lange ab, bis sie aufgrund der zunehmenden Exazerbation der Erkrankung nicht mehr vermeidbar ist. Bei der Schließung der Depressionsstation erklärten einzelne Patienten, sie wären viel früher gekommen, wenn sie das Angebot der ambulanten Akutbehandlung verfügbar gehabt hätten. Für diese Patientengruppe erscheint es denkbar, langfristig erhebliche Kosten zu sparen, wenn eine ambulante Akutbehandlung die Betroffenen wohnortnah, frühzeitig und niederschwellig erreichen kann.

Das vorliegende Projekt würde sicherlich dadurch erleichtert, dass die Versicherten aller Krankenkassen eingeschlossen werden könnten. Die Führung einer psychiatrischen Klinik mit häufigen Notfall- und Zwangseinweisungen stellt eine besondere Herausforderung dar. Diese Aufgabe wird durch die Notwendigkeit, die Prozessabläufe abhängig von der Krankenversicherung des Patienten gestalten zu müssen, nicht unbedingt vereinfacht. Hinzu kommt, dass es die meisten Patienten als befremdlich erleben, dass sie den Zugang $\mathrm{zu}$ einem besonderen Therapieangebot nur über den stationären Aufenthalt erhalten können, während Versicherte der Techniker Krankenkasse und der AOK Hessen im Rahmen der ambulanten Akutbehandlung teilnehmen.

Unabhängig von den wirtschaftlichen Effekten bei verschiedenen Patientengruppen sind von einer ambulanten Akutbehandlung Effizienzgewinne im Bereich der Krankenhausinfrastruktur dadurch zu erwarten, dass diese Versorgungsformen bei anhaltend wachsenden Patientenzahlen ohne einen proportionalen Ausbau von stationären Betten auskommen können.

\subsection{Netzwerk von OVP Kliniken}

Für die weitere Entwicklung des OVPProjektes ist die Ausweitung auf andere Kliniken und Versorgungsregionen ein wichtiger Schritt. Die Vorzüge des Projektes sind so weitreichend, dass sich bundesweit eine Reihe von Kliniken dafür interessiert. Um die Hürden der Einschreibung in einen IV-Vertrag zu beseitigen, ist dabei die Weiterentwicklung des OVP-Projektes zu einem Modellprojekt nach $\mathbb{S} 64 \mathrm{~b}$ SGB V geplant. Für die beteiligten Kliniken bietet sich dabei ein Netzwerk für eine gemeinsame vergleichende Versorgungsforschung an.

\section{Literatur}

Lehmann G., Reddig V., Mendel M., ThiexKreye M., Schillen T., Fischer S. (2011) Die finanziellen Anreize werden sich ändern. f\&w 4/2011, 389-392 